\title{
Analysing Dynamical Systems Towards Computing Complete Lyapunov Functions
}

\author{
Carlos Argáez ${ }^{1}$, Peter Giesl ${ }^{2}$ and Sigurdur Hafstein ${ }^{1}$ \\ ${ }^{1}$ Faculty of Physical Sciences, University of Iceland, 107 Reykjavik, Iceland \\ ${ }^{2}$ Department of Mathematics, University of Sussex, Falmer BN1 9QH, U.K.
}

Keywords: Dynamical System, Complete Lyapunov Function, Meshless Collocation, Radial Basis Functions.

\begin{abstract}
Ordinary differential equations arise in a variety of applications, including e.g. climate systems, and can exhibit complicated dynamical behaviour. Complete Lyapunov functions can capture this behaviour by dividing the phase space into the chain-recurrent set, determining the long-time behaviour, and the transient part, where solutions pass through. In this paper, we present an algorithm to construct complete Lyapunov functions. It is based on mesh-free numerical approximation and uses the failure of convergence in certain areas to determine the chain-recurrent set. The algorithm is applied to three examples and is able to determine attractors and repellers, including periodic orbits and homoclinic orbits.
\end{abstract}

\section{INTRODUCTION}

In this paper, we describe a new algorithm to analyse the behaviour of a dynamical system. Let us consider a general autonomous ordinary differential equation (ODE) $\dot{\mathbf{x}}=\mathbf{f}(\mathbf{x})$, where $\mathbf{x} \in \mathbb{R}^{n}$.

A classical (strict) Lyapunov function (Lyapunov, 1992) is a scalar-valued function that can be used to analyse the basin of attraction of one attractor such as an equilibrium or a periodic orbit. It attains its minimum at the attractor, and is otherwise strictly decreasing along solutions of the ODE.

A generalization of this idea is the notion of a complete Lyapunov function (Conley, 1978a; Conley, 1988; Hurley, 1995; Hurley, 1998), which characterizes the complete behaviour of the dynamical system. It is a scalar-valued function $V: \mathbb{R}^{n} \rightarrow \mathbb{R}$, defined on the whole phase space, not just in a neighborhood of one particular attractor. It is non-increasing along solutions of the ODE. The phase space can be divided into the area where the complete Lyapunov function strictly decreases along solution trajectories and the one where it is constant along solution trajectories. The area where the complete Lyapunov function is strictly decreasing along solution trajectories characterizes the gradient-like flow. There solutions pass through and the larger this area is, the more information is obtained from the complete Lyapunov function. Note that, by definition, the complete Lyapunov function needs to be constant along solution trajectories on each transitive component of the chainrecurrent set.

Note that there are other methods to analyse the general behaviour of dynamical systems: The direct simulation of solutions with many different initial conditions, however, is costly and can only give limited information about the general behaviour of the system, unless estimates are available, e.g. when shadowing solutions. More sophisticated methods include the computation of invariant manifolds, forming the boundaries of basins of attraction of attractors (Krauskopf et al., 2005); here, additional analysis of the parts with gradient-like flow is necessary. The cell mapping approach (Hsu, 1987) or set oriented methods (Dellnitz and Junge, 2002) divide the phase space into cells and compute the dynamics between these cells, see also for example (Osipenko, 2007); these ideas have also been used to compute complete Lyapunov functions, see Section 2.1.

In this paper we focus on a new algorithm of tackling a mathematical problem numerically. Inspired by a method to compute classical Lyapunov functions for an equilibrium, we approximate the solution of $V^{\prime}(\mathbf{x})=-1$, where $V^{\prime}(\mathbf{x})=\nabla V(\mathbf{x}) \cdot \mathbf{f}(\mathbf{x})$ denotes the orbital derivative, the derivative along solutions of the ODE. We use mesh-free collocation with Radial Basis Function to approximately solve this partial differential equation (PDE): we choose a finite set of collocation points $X$ and compute an approximation $v$ to $V$ which solves the PDE in all collocation points. 
Note, however, that the PDE cannot be fulfilled at all points of the chain-recurrent set, such as an equilibrium or periodic orbit. We use the failure of the method to approximate in certain areas to identify these areas. We split the collocation points $X$ into a set $X^{0}$, where the approximation fails, and $X^{-}$, where it works well. A complete Lyapunov function should be constant in $X^{0}$, so in the next step we solve the PDE $V^{\prime}(\mathbf{x})=0$ for all $\mathbf{x} \in X^{0}$ and $V^{\prime}(\mathbf{x})=-1$ for all $\mathbf{x} \in X^{-}$, which is an approximation of a complete Lyapunov function.

The computed function $v$ gives us the following information about the ODE under consideration: the set $X^{0}$, where $v^{\prime}(\mathbf{x}) \approx 0$ approximates the chainrecurrent set, including equilibria, periodic orbits and homoclinic orbits, while the set $X^{-}$, where $v^{\prime}(\mathbf{x}) \approx-1$ approximates the area with gradient-like flow, where solutions pass through. The function $v$, through its level sets, gives information about the stability and attraction properties: minima of $v$ correspond to attractors, while maxima represent repellers.

Let us give an overview over the paper: in Section 2 we discuss complete Lyapunov functions as well as mesh-free collocation to approximate solutions of a general linear PDE. In Section 3 we present our algorithm to compute a complete Lyapunov function. In Sections 4 and 5 we apply the method to three examples and discuss the results in detail.

\section{PRELIMINARIES}

\subsection{Complete Lyapunov Functions}

We will consider a general autonomous ODE

$$
\dot{\mathbf{x}}=\mathbf{f}(\mathbf{x}), \text { where } \mathbf{x} \in \mathbb{R}^{n} .
$$

A complete Lyapunov function (Conley, 1978b; Conley, 1978a) is a continuous function $V: \mathbb{R}^{n} \rightarrow \mathbb{R}$ which is constant on the chain-recurrent set, including local attractors and repellers, and decreasing elsewhere. In contrast to classical Lyapunov functions (Lyapunov, 1992), which are defined on the basin of attraction of just one attractor, a complete Lyapunov function characterizes the flow on the whole phase space and distinguishes between the chain-recurrent set and the gradient-like flow. Thus, it captures the long-term behaviour of the system.

Conley (Conley, 1978b; Conley, 1978a) proved the existence of complete Lyapunov functions for dynamical systems on a compact metric space. The idea is to consider corresponding attractor-repeller pairs and to construct a function which is 1 on the repeller, 0 on the attractor and decreasing in between. Then these functions are summed up over all attractorrepeller pairs. This was generalized to more general spaces by Hurley (Hurley, 1992; Hurley, 1995; Hurley, 1998).

The smaller the part of the phase space where the complete Lyapunov function is constant, the more information is provided by a complete Lyapunov function. There exists a complete Lyapunov function which is only constant on the generalized chainrecurrent set (Auslander, 1964), thus providing further information about the system as the generalized chain-recurrent set is a subset of the chain-recurrent set.

In (Kalies et al., 2005; Ban and Kalies, 2006; Goullet et al., 2015) a computational approach to construct complete Lyapunov functions was considered. A discrete-time system was given by the time$T$ map and the phase space was subdivided into cells and the dynamics between them computed through an induced multivalued map using the computer package GAIO (Dellnitz et al., 2001). An approximate complete Lyapunov function is then computed using graph algorithms (Ban and Kalies, 2006). This approach requires a high number of cells even for low dimensions. We will use a different methodology, inspired by the construction of classical Lyapunov functions, which is faster and works well in higher dimensions. In (Björnsson et al., 2014a), the approach of (Ban and Kalies, 2006) is compared to the RBF method for equilibria (see below) for one particular example; here, the method of (Ban and Kalies, 2006) works well only on the chain-recurrent set, while the RBF method is very efficient on the gradient-like part.

In (Björnsson et al., 2015), a complete Lyapunov is constructed as a continuous piecewise affine (CPA) function, affine on a fixed simplicial complex. However, it is assumed that information about local attractors is a available, while the proposed method in this paper does not require any information about the system under consideration.

\subsection{Mesh-free Collocation}

For classical Lyapunov functions, several numerical construction methods have recently been proposed, e.g. (Johansson, 1999; Johansen, 2000; Marinósson, 2002; Giesl, 2007; Hafstein, 2007; Björnsson et al., 2014b; Kamyar and Peet, 2015; Anderson and Papachristodoulou, 2015; Doban, 2016; Doban and Lazar, 2016) see also the recent review (Giesl and Hafstein, 2015). Our algorithm will be based on the RBF (Radial Basis Function) method, a special case of mesh-free collocation, which approximates the solution of a linear PDE, specifying the orbital deriva- 
tive.

Mesh-free methods, particularly based upon Radial Basis Functions, provide a powerful tool for solving generalized interpolation problems efficiently. We assume that the target function belongs to a Hilbert space $H$ of continuous functions (often a Sobolev space) with reproducing kernel $\varphi: \mathbb{R}^{n} \times \mathbb{R}^{n} \rightarrow \mathbb{R}$, given by a suitable Radial Basis Function $\Phi$ through $\varphi(\mathbf{x}, \mathbf{y}):=\Phi(\mathbf{x}-\mathbf{y})$, where $\Phi(\mathbf{x})=\psi(\|\mathbf{x}\|)$ is a radial function. Examples for Radial Basis Functions include the Gaussians, multiquadrics and inverse multiquadrics; we, however, will use the compactly supported Wendland functions in this paper, which will be defined below.

We assume that the information $r_{1}, \ldots, r_{N} \in \mathbb{R}$ of a target function $V \in H$ generated by $N$ linearly independent functionals $\lambda_{j} \in H^{*}$ is known. The optimal reconstruction of the function $V$ is the solution of the minimization problem $\min \left\{\|v\|_{H}: \lambda_{j}(v)=r_{j}, 1 \leq j \leq\right.$ $N\}$. It is well-known (Wendland, 2005) that the solution can be written as $v(\mathbf{x})=\sum_{j=1}^{N} \beta_{j} \lambda_{j}^{\mathbf{y}} \varphi(\mathbf{x}, \mathbf{y})$, where the coefficients $\beta_{j}$ are determined by the interpolation conditions $\lambda_{j}(v)=r_{j}, 1 \leq j \leq N$.

In our case, we consider the PDE $V^{\prime}(\mathbf{x})=g(\mathbf{x})$, where $g(\mathbf{x})$ is a given function. We choose $N$ points $\mathbf{x}_{1}, \ldots, \mathbf{x}_{N} \in \mathbb{R}^{n}$ of the phase space and define functionals $\lambda_{j}(v):=\left(\delta_{\mathbf{x}_{j}} \circ L\right)^{\mathbf{x}} v=v^{\prime}\left(\mathbf{x}_{j}\right)=\nabla v\left(\mathbf{x}_{j}\right) \cdot \mathbf{f}\left(\mathbf{x}_{j}\right)$, where $L$ denotes the linear operator of the orbital derivative $L V(\mathbf{x})=V^{\prime}(\mathbf{x})$ and $\delta$ is Dirac's delta distribution. The right-hand sides are $V_{j}^{\prime}=g\left(\mathbf{x}_{j}\right)$ for all $1 \leq j \leq N$. The approximation is then

$$
v(\mathbf{x})=\sum_{j=1}^{N} \beta_{j}\left(\delta_{\mathbf{x}_{j}} \circ L\right)^{\mathbf{y}} \Phi(\mathbf{x}-\mathbf{y}),
$$

where $\Phi$ is a positive definite Radial Basis Function, and the coefficients $\beta_{j} \in \mathbb{R}$ can be calculated by solving a system of $N$ linear equations. A crucial ingredient is the knowledge on the behaviour of the error function $\left|V^{\prime}(\mathbf{x})-v^{\prime}(\mathbf{x})\right|$ in terms of the so-called fill distance which measures how dense the points $\left\{\mathbf{x}_{1}, \ldots, \mathbf{x}_{N}\right\}$ are, since it gives information when the approximate solution indeed becomes a Lyapunov function, i.e. has a negative orbital derivative. Such error estimates were derived, for example in (Giesl, 2007; Giesl and Wendland, 2007), see also (Narcowich et al., 2005; Wendland, 2005).

The advantage of mesh-free collocation over other methods for solving PDEs is that scattered points can be added to improve the approximation, no triangulation of the phase space is necessary, the approximating function is smooth and the method works in any dimension.

In this paper, we use Wendland functions (Wendland, 1998) as Radial Basis Functions through $\psi(\mathbf{x}):=\psi_{l, k}(c\|\mathbf{x}\|)$, where $c>0, k \in \mathbb{N}$ is a smoothness parameter and $l=\left\lfloor\frac{n}{2}\right\rfloor+k+1$. Wendland functions are positive definite functions with compact support, which are polynomials on their support; the corresponding Reproducing Kernel Hilbert Space is norm-equivalent to the Sobolev space $W_{2}^{k+(n+1) / 2}\left(\mathbb{R}^{n}\right)$. They are defined by recursion: for $l \in \mathbb{N}, k \in \mathbb{N}_{0}$ we define

$$
\begin{array}{r}
\psi_{l, 0}(r)=(1-r)_{+}^{l} \\
\psi_{l, k+1}(r)=\int_{r}^{1} t \psi_{l, k}(t) \mathrm{d} t
\end{array}
$$

for $r \in \mathbb{R}_{0}^{+}$, where $x_{+}=x$ for $x \geq 0$ and $x_{+}=0$ for $x<0$.

As collocation points $X \subset \mathbb{R}^{n}$ we use a hexagonal grid with $\alpha \in \mathbb{R}^{+}$constructed according to

$$
\begin{gathered}
\left\{\alpha \sum_{k=1}^{n} i_{k} w_{k}: i_{k} \in \mathbb{Z}\right\}, \text { where } \\
w_{1}=\left(2 e_{1}, 0,0, \ldots, 0\right) \\
w_{2}=\left(e_{1}, 3 e_{2}, 0, \ldots, 0\right) \\
\vdots \quad \vdots \\
w_{n}=\left(e_{1}, e_{2}, e_{3}, \ldots,(n+1) e_{n}\right) \text { and } \\
e_{k}=\sqrt{\frac{1}{2 k(k+1)}}, k \in \mathbb{N} .
\end{gathered}
$$

We set $\psi_{0}(r):=\psi_{l, k}(c r)$ with positive constant $c$ and define recursively $\psi_{i}(r)=\frac{1}{r} \frac{d \psi_{i-1}}{d r}(r)$ for $i=1,2$ and $r>0$. The explicit formulas for $v$ and its orbital derivative are

$$
\begin{aligned}
v(\mathbf{x}) & =\sum_{j=1}^{N} \beta_{j}\left\langle\mathbf{x}_{j}-\mathbf{x}, \mathbf{f}\left(\mathbf{x}_{j}\right)\right\rangle \psi_{1}\left(\left\|\mathbf{x}-\mathbf{x}_{j}\right\|\right) \\
v^{\prime}(\mathbf{x})= & \sum_{j=1}^{N} \beta_{j}\left[-\psi_{1}\left(\left\|\mathbf{x}-\mathbf{x}_{j}\right\|\right)\left\langle\mathbf{f}(\mathbf{x}), \mathbf{f}\left(\mathbf{x}_{j}\right)\right\rangle\right. \\
& \left.+\psi_{2}\left(\left\|\mathbf{x}-\mathbf{x}_{j}\right\|\right)\left\langle\mathbf{x}-\mathbf{x}_{j}, \mathbf{f}(\mathbf{x})\right\rangle \cdot\left\langle\mathbf{x}_{j}-\mathbf{x}, \mathbf{f}\left(\mathbf{x}_{j}\right)\right\rangle\right]
\end{aligned}
$$

where $\beta$ is the solution of $A \cdot \beta=\mathbf{r}, r_{j}=V^{\prime}\left(\mathbf{x}_{j}\right)$ and $A$ is the $N \times N$ matrix with entries

$$
\begin{aligned}
a_{i j}= & \psi_{2}\left(\left\|\mathbf{x}_{i}-\mathbf{x}_{j}\right\|\right)\left\langle\mathbf{x}_{i}-\mathbf{x}_{j}, \mathbf{f}\left(\mathbf{x}_{i}\right)\right\rangle\left\langle\mathbf{x}_{j}-\mathbf{x}_{i}, \mathbf{f}\left(\mathbf{x}_{j}\right)\right\rangle \\
& -\psi_{1}\left(\left\|\mathbf{x}_{i}-\mathbf{x}_{j}\right\|\right)\left\langle\mathbf{f}\left(\mathbf{x}_{i}\right), \mathbf{f}\left(\mathbf{x}_{j}\right)\right\rangle
\end{aligned}
$$

for $i \neq j$ and

$$
a_{i i}=-\psi_{1}(0)\left\|\mathbf{f}\left(\mathbf{x}_{i}\right)\right\|^{2} .
$$

More detailed explanations on this construction are given in (Giesl, 2007, Chapter 3).

If no collocation point $\mathbf{x}_{j}$ is an equilibrium for the system, i.e. $\mathbf{f}\left(\mathbf{x}_{j}\right) \neq \mathbf{0}$ for all $j$, then the matrix $A$ is 
positive definite and the system of equations $A \beta=\mathbf{r}$ has a unique solution. Note that this holds true independent of whether the underlying discretized PDE has a solution or not, while the error estimates are only available if the PDE has a solution.

\section{ALGORITHM}

Starting with scattered collocation points $X$, we solve the equation $V^{\prime}(\mathbf{x})=-1$, where $V^{\prime}(\mathbf{x}):=\nabla V(\mathbf{x}) \cdot \mathbf{f}(\mathbf{x})$ denotes the orbital derivative, the derivative along solutions of (1). Note that the equation $V^{\prime}(\mathbf{x})=-1$ does not have a solution on chain-recurrent sets in general; e.g. along a periodic orbit, the orbital derivative must integrate to 0 . However, as mentioned above, we can still compute a (unique) approximation by the method described in Section 2.2.

In the next step we check for each collocation point $\mathbf{x}_{j}$ in $X$ whether the approximation was poor (then $\mathbf{x}_{j} \in X^{0}$ ) or good (then $\mathbf{x}_{j} \in X^{-}$). Then we approximate the solution of the new problem $V^{\prime}(\mathbf{x})=$ -1 for $\mathbf{x} \in X^{-}$and $V^{\prime}(\mathbf{x})=0$ for $\mathbf{x} \in X^{0}$; the set $X^{0}$ indicates the (generalized) chain-recurrent set.

To determine whether the approximation was poor or good, we evaluate $v^{\prime}(\mathbf{x})$ for test points $\mathbf{x}$ around each collocation point $\mathbf{x}_{j}$ - for a good approximation we expect $v^{\prime}(\mathbf{x}) \approx-1$. In view of our goal to compute a complete Lyapunov function, we classify collocation points as poor if the orbital derivative near them is larger than 0 or a chosen critical value, i.e. $v^{\prime}(\mathbf{x})>\gamma$, for certain points $\mathbf{x}$ near the collocation point $\mathbf{x}_{j}$. As points to check near a collocation point $\mathbf{x}_{j}$ we choose points on two circumferences around each collocation point $\mathbf{x}_{j}$. They are distributed around each collocation point and we place 64 checking points per each collocation point. In particular, in $\mathbb{R}^{2}$, for a collocation point $\mathbf{x}_{j} \in \mathbb{R}^{2}$, we use the following checking points $Y_{\mathbf{x}_{j}}$ :

$$
\begin{aligned}
& \mathbf{x}_{j} \pm r \alpha(\cos (\theta),-\sin (\theta)) \\
& \mathbf{x}_{j} \pm r \alpha(\sin (\theta), \cos (\theta)) \\
& \mathbf{x}_{j} \pm \frac{r}{2} \alpha *(\cos (\theta), \sin (\theta)) \\
& \mathbf{x}_{j} \pm \frac{r}{2} \alpha *(\cos (\theta),-\sin (\theta))
\end{aligned}
$$

where $r$ is a scaling parameter and $\theta=$ $0.0,11.25,22.5,45,56.25,67.5,75$ and 105 degrees; this is shown in Figure 1.

The algorithm can be summarized as follows.

1. Create the collocation points $X$ and compute the approximate solution $v$ of $V^{\prime}(\mathbf{x})=-1$

2. For each collocation point $\mathbf{x}_{j}$, compute $v^{\prime}(\mathbf{x})$ : if $v^{\prime}(\mathbf{x})>\gamma$ for a point $\mathbf{x} \in Y_{\mathbf{x}_{j}}$, then $\mathbf{x}_{j} \in X^{0}$, otherwise $\mathbf{x}_{j} \in X^{-}$, where $\gamma \leq 0$ is a chosen critical value

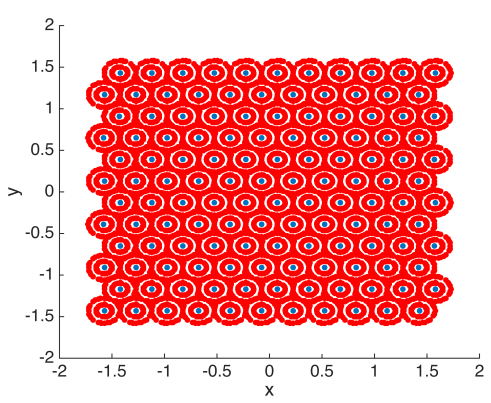

Figure 1: The figure shows the collocation points (blue) and the checking points (red) around each collocation point. The collocations points are used to construct the Lyapunov function and the checking points are used to evaluate the orbital derivative of the complete Lyapunov function.

3. Compute the approximate solution $v$ of $V^{\prime}(\mathbf{x})=$ -1 for $\mathbf{x} \in X^{-}$and $V^{\prime}(\mathbf{x})=0$ for $\mathbf{x} \in X^{0}$

4. Repeat steps 2. and 3. until no more points are added to $X^{0}$

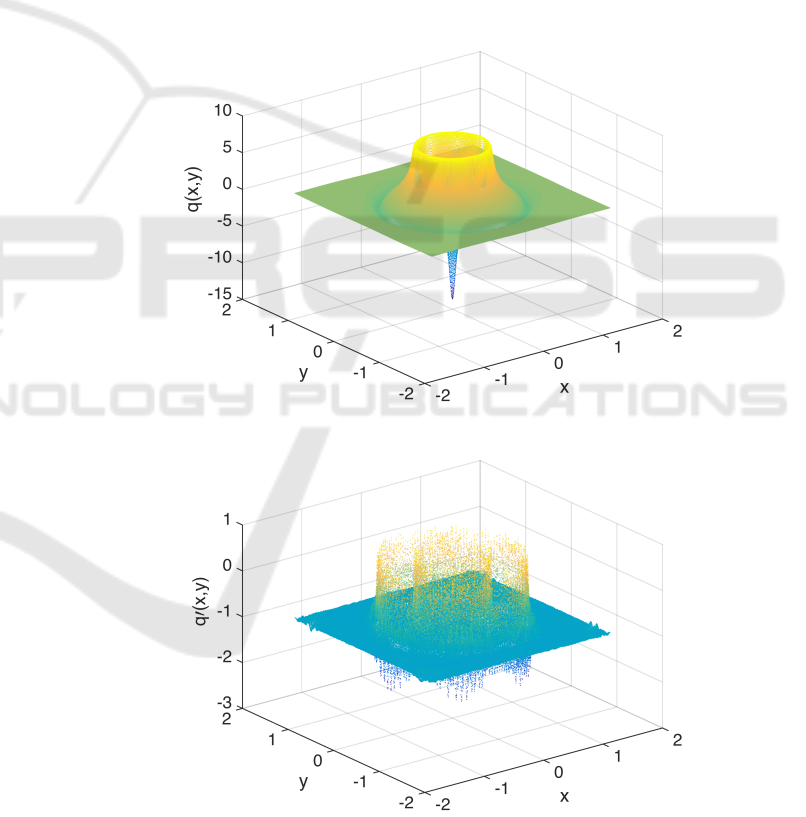

Figure 2: Lyapunov function for (4). The upper figure shows the approximated complete Lyapunov function. The lower figure shows its orbital derivative. The minima in the upper figure correspond to the asymptotically stable equilibrium and periodic orbit with radius 1 , while the maximum is attained at the repelling periodic orbit of radius $1 / 2$. The orbital derivative fails to be negative on the periodic orbits and the equilibrium. 

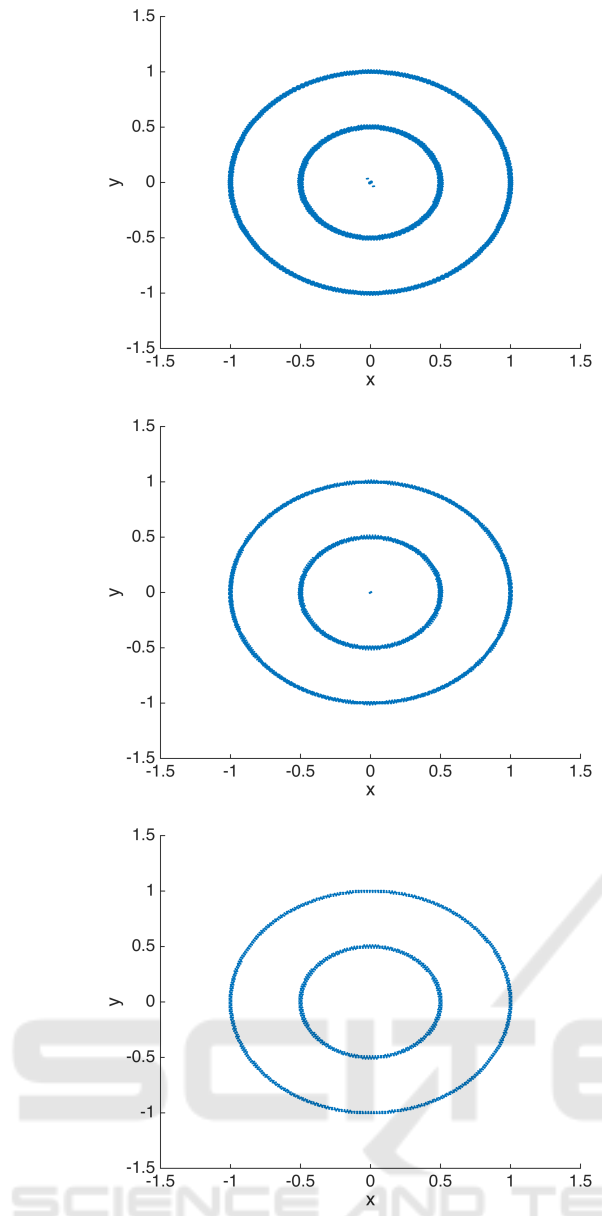

Figure 3: The figures show the checking points $\mathbf{x}$ around each collocation point, where $v^{\prime}(\mathbf{x})>\gamma$ for different values of $\gamma$. Upper: $\gamma=-0.5$. Middle: $\gamma=-0.25$. Lower: $\gamma=0$. In all cases, $\alpha=0.02, r=0.5$.

\section{EXAMPLES}

In the following we apply the method to three examples and then analyse the behaviour of the method with respect to certain parameters. In this section we only consider the first step of the method, and we will discuss the iterations in Section 5. Note that in all examples we use the notation $\mathbf{x}=(x, y)$.

\subsection{Two Circular Periodic Orbits}

We consider system (1) with right-hand side

$$
\mathbf{f}(x, y)=\left(\begin{array}{l}
-x\left(x^{2}+y^{2}-1 / 4\right)\left(x^{2}+y^{2}-1\right)-y \\
-y\left(x^{2}+y^{2}-1 / 4\right)\left(x^{2}+y^{2}-1\right)+x
\end{array}\right),
$$

which has an asymptotically stable equilibrium at the origin, an asymptotically stable periodic orbit with radius 1 , and a repelling periodic orbit with radius $1 / 2$.
To compute the Lyapunov function with our method we used $\psi_{5,3}$ as Wendland function with $c=$ 1. The collocation points were set in $(-1.5,1.5) \times$ $(-1.5,1.5) \subset \mathbb{R}^{2}$ and we used $\alpha=0.02$.

In Figure 2 we see how the derivative fails to be negative in both periodic orbits and the equilibrium. In the next step of the algorithm, we use points around each collocation points to check whether the orbital derivative is above the critical value $\gamma$. Figure 3 shows the results for different values of $\gamma$, namely $\gamma=0,-0.25,-0.5$ and a fixed scaling parameter $r=$ 0.5 for (3).

Clearly, $\gamma=0$ is not enough to find all failing points, since some chain-recurrent sets, such as the equilibrium at the origin, are not detected.

\subsection{Van Der Pol Oscillator System}

We consider system (1) with right-hand side

$$
\mathbf{f}(x, y)=\left(\begin{array}{c}
y \\
\left(1-x^{2}\right) y-x
\end{array}\right) .
$$

System (5) is the two dimensional form of the Van der Pol oscillator. This describes the behaviour of a nonconservative oscillator reacting to a non-linear damping. The origin is an unstable focus, and the system has an asymptotically stable periodic orbit.

We used our method with the Wendland function $\psi_{4,2}$ and $c=1$. The collocation points were set in $(-4,4) \times(-4,4) \subset \mathbb{R}^{2}$ with $\alpha=0.1$.

In Figure 4 we see how the derivative fails to be negative at the points of the periodic orbit. In Figure 5 we show the points in $X^{0}$ for three critical values $\gamma=0,-0.25,-0.5$ and a fixed scaling parameter $r=0.5$.

For $\gamma=0$ and $\gamma=-0.25$ we do not detect all points of the periodic orbit, but we can already see its shape (see Figure 5), while for $\gamma=-0.5$ the whole periodic orbit is covered. However, we obtain points in the gradient-like flow where the orbital derivative fails to be negative, and thus overestimate the chain-recurrent set.

\subsection{Homoclinic Orbit}

We consider system (1) with right-hand side $\mathbf{f}(x, y)=$

$$
\left(\begin{array}{l}
x\left(1-x^{2}-y^{2}\right)-y\left((x-1)^{2}+\left(x^{2}+y^{2}-1\right)^{2}\right) \\
y\left(1-x^{2}-y^{2}\right)+x\left((x-1)^{2}+\left(x^{2}+y^{2}-1\right)^{2}\right)
\end{array}\right) .
$$

This system has an unstable focus at the origin and an asymptotically stable homoclinic orbit at a circle centered at the origin and with radius 1 . 

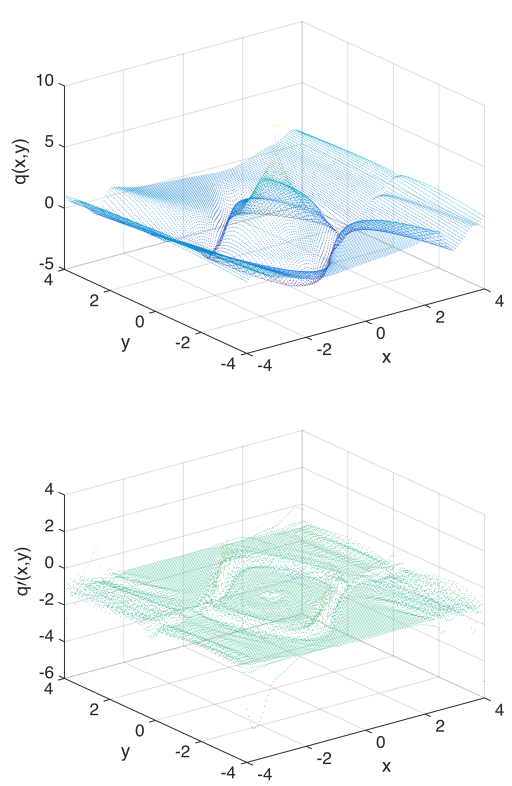

Figure 4: Approximated complete Lyapunov function for (5). The upper figure shows the complete Lyapunov function; the minimum corresponds to the asymptotically stable periodic orbit and the maximum to the unstable equilibrium at the origin. The lower figure shows the orbital derivative of the complete Lyapunov function. The collocation points in both cases have $\alpha=0.1$. The lower figure is able to detect the periodic orbit.

We use our method with the Wendland function $\psi_{4,2}$ and $c=1$. The collocation points were set in $(-1.5,1.5) \times(-1.5,1.5) \subset \mathbb{R}^{2}$ with $\alpha=0.02$.

The method detects the points of the homoclinic orbit well, but has problems at the unstable equilibrium at $(1,0)$, corresponding to the homoclinic orbit. Here, the approximation also fails at points which belong to the gradient-like flow.

In Figure 7 we see how the derivative fails to be negative at the points of the homoclinic orbit, and also beyond. We show the points in $X^{0}$ for three critical values $\gamma=0,-0.25,-0.5$ and a fixed scaling radio $r=0.5$.

\subsection{Dependence on the Collocation Grid and the Verification Points}

A very interesting behaviour is observed by varying the density of the collocation grid. A natural way to vary it is by changing the value of the parameter $\alpha$ in (3): the larger the $\alpha$ the fewer collocation points we have for a defined area.

In Figure 8 we can observe how our algorithm converges to the chain-recurrent set for example (4). We observe that in order to be able to detect the dy-
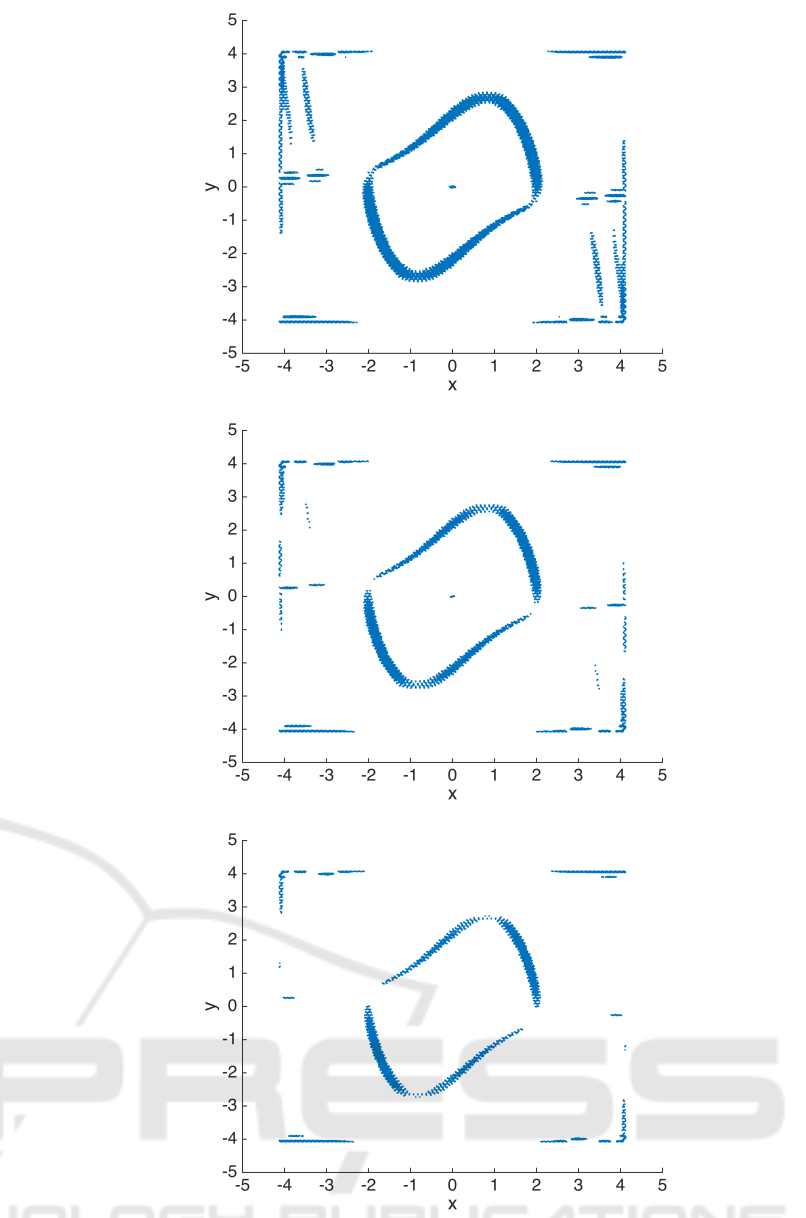

Figure 5: The figures show the points $\mathbf{x}$ around each collocation point, where $v^{\prime}(\mathbf{x})>\gamma$ for different values of $\gamma$. Upper: $\gamma=-0.5$. Middle: $\gamma=-0.25$. Lower: $\gamma=0$. In all cases, $\alpha=0.1, r=0.5$.

namical behaviour, the collocation grid needs to be sufficiently dense. This corresponds to error estimates between the solution of a PDE and its approximation using mesh-free collocation which proves that the error is bounded by a constant times the fill distance, measuring the density of the collocation points. In our case, however, the PDE does not have a solution and thus these error estimates do not apply.

In Figure 9 we consider example (6) and vary the radius $r$ for the checking points, used to determine poor and good collocation points. We are only able to detect the whole homoclinic orbit with a sufficiently large radius. This is to be expected, since at the collocation points we have $v^{\prime}(\mathbf{x})=-1$ and since $v^{\prime}$ is continuous it will be negative close enough to the collocation points. 

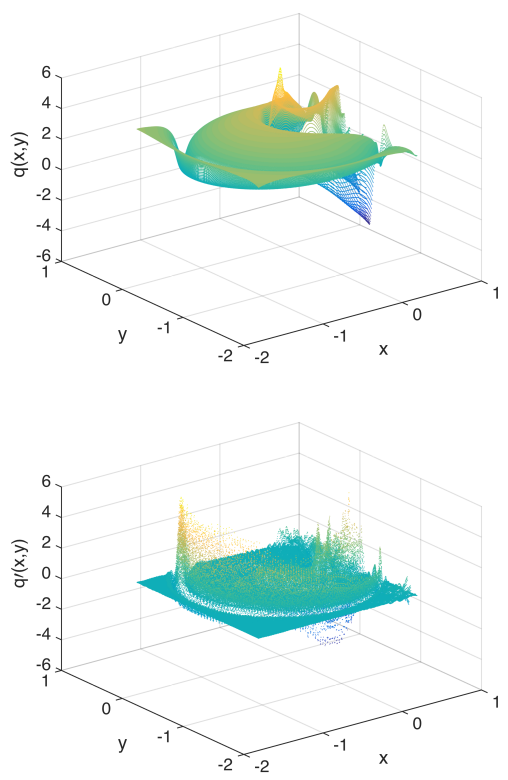
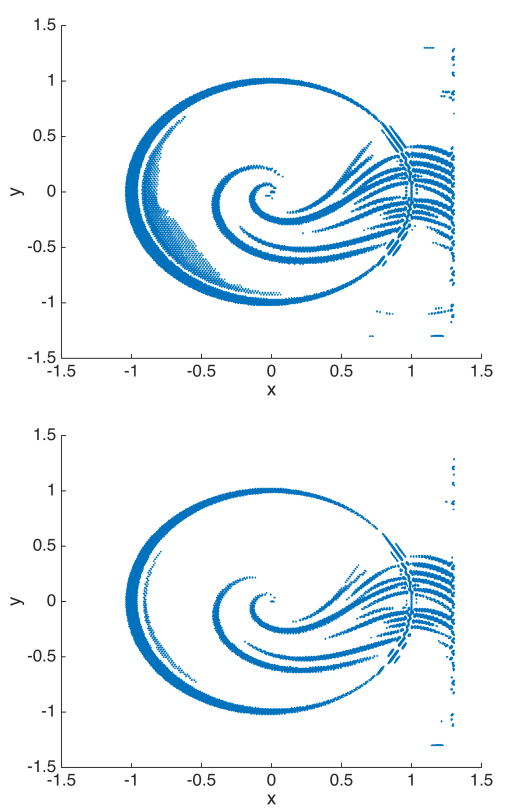

Figure 6: Approximated complete Lyapunov function for (6). The upper figure shows the approximated complete Lyapunov function; the homoclinic orbit corresponds to the minimum, while the origin is a maximum. The lower figure shows its orbital derivative. The collocation points in both cases have $\alpha=0.02$.

\section{ITERATION STEPS OF THE ALGORITHM}

After splitting the set of collocation points $X$ into the set $X^{0}$, where the approximation is poor, and the remaining points $X^{-}$, in the next step, our method will solve the problem $V^{\prime}(\mathbf{x})=0$ for $\mathbf{x} \in X^{0}$ and $V^{\prime}(\mathbf{x})=$ -1 for $\mathbf{x} \in X^{-}$. This is done by solving $A \beta=\mathbf{r}$ with an updated right-hand-side $\mathbf{r}$ and we look again for collocation points where the approximation is poor. This procedure should then be iterated until no more collocation points are added to $X^{0}$.

For the Van der Pol system (5) the results of this procedure with $\gamma=-0.25$ and $\alpha=0.1$ can be seen in Figures 10 to 12 . Figure 10 shows the complete Lyapunov $v$ for each step, which does not change significantly after the first step; observe, that the range of $v$ is slightly reduced. Figure 11 shows its orbital derivative; again, there is no significant change after the first step, only the range of $v^{\prime}$ is slightly reduced and the values near the chain-recurrent set are now 0 rather than different values above the critical value.

Figure 12, finally, displays the set of checking points where the orbital derivative is larger than the critical value $\gamma=-0.25$; these points should approximate the chain-recurrent set, in this case the unstable equilibrium at the origin and the asymptotically sta-

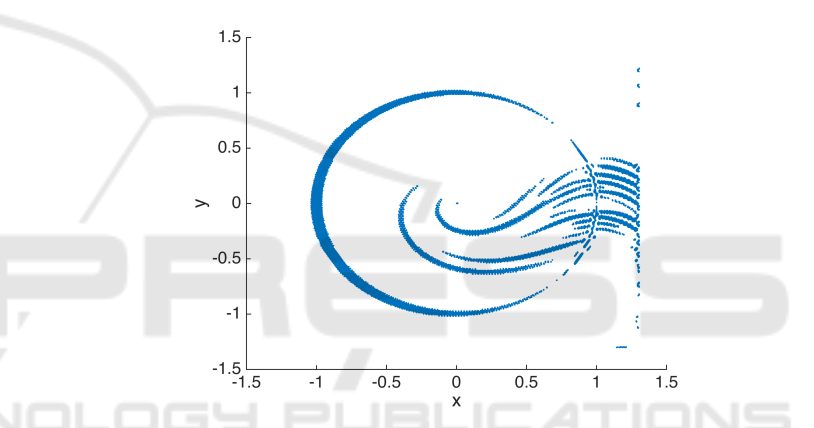

Figure 7: The figures show the points $\mathbf{x}$ around each collocation point, where $v^{\prime}(\mathbf{x})>\gamma$ for different values of $\gamma$. Upper: $\gamma=-0.5$. Middle: $\gamma=-0.25$. Lower: $\gamma=0$. In all cases, $\alpha=0.02, r=0.5$.

ble periodic orbit. We observe that the set of points increases and nearly covers the whole periodic orbit. However, collocation points away from the chainrecurrent set also are included, mainly at the boundary of the domain under consideration as the values of $v^{\prime}(\mathbf{x})$ become more extreme.

After the first step there is no significant change in the figures, although a close study reveals that the algorithm still adds points, see Table 1 . There are boundary effects, such that points near the boundary of the considered domain do not behave as expected. We are currently studying these phenomena and expect that a more elaborate way of fixing the values of $v^{\prime}$ at the collocation points will improve the performance of the method.

For all examples (4) to (6) we show a table of how many points we find by iterating this procedure. The corresponding parameters are given in Table 2.

In Table 1 the number of total collocation points and 

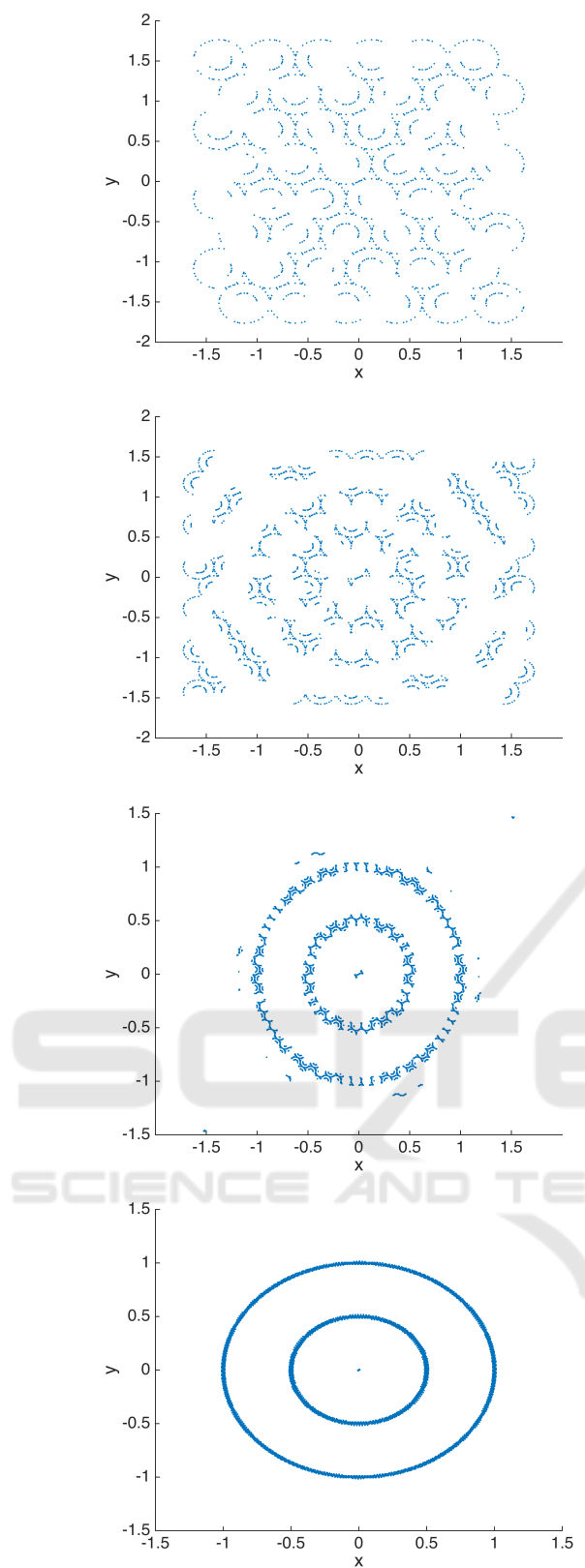

Figure 8: Example (4) with collocation grids of varying density. Upper $\alpha=0.5$. Middle up $\alpha=0.3$. Middle down $\alpha=0.1$. Lower $\alpha=0.02$. The radii for the test circles for all of them were set to $r=0.5$ and the critical value was $\gamma=-0.25$.

the total number of points in $X^{0}$ with poor approximation in each step iteration are shown. For system (4) we see that after the fourth step no more points are added to $X^{0}$, while for the other two examples the method keeps adding points.
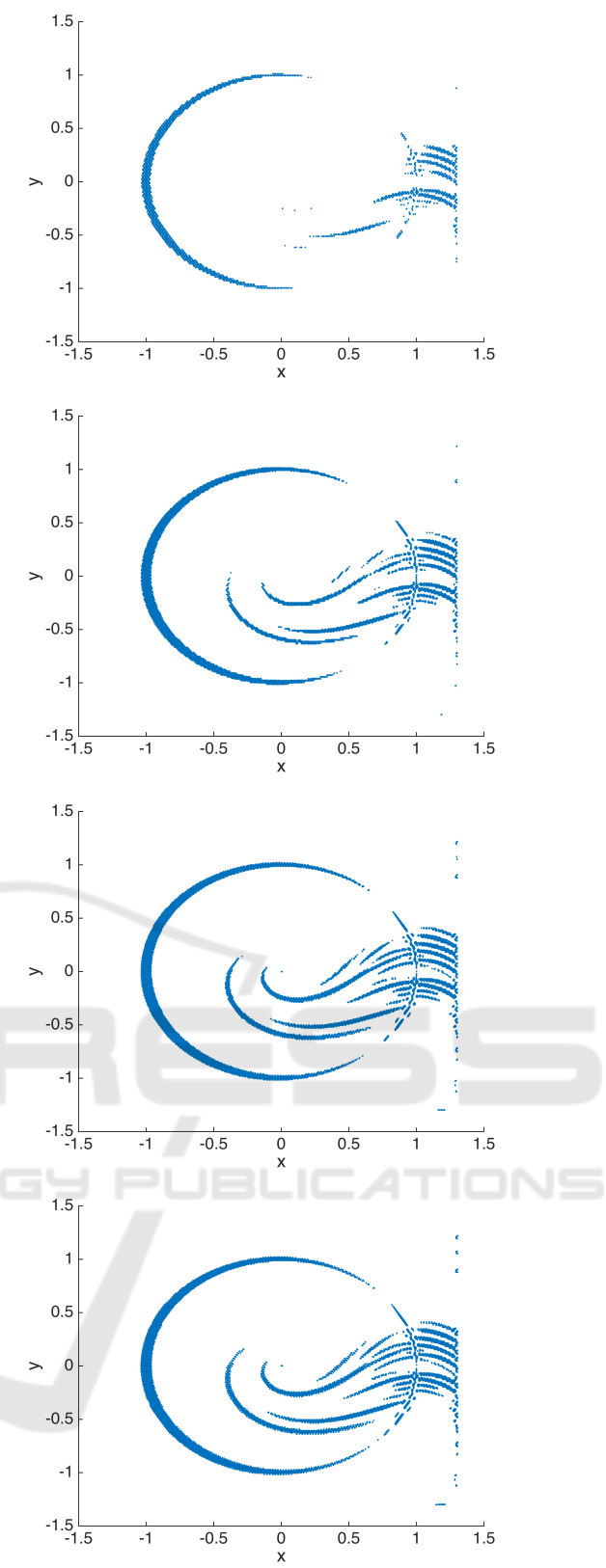

Figure 9: We consider example (6) with fixed $\alpha=0.02$ fixed critical value $\gamma=0.0$ and varying $r$. Upper $r=0.2$. Middle up $r=0.3$. Middle down $r=0.4$. Lower $r=0.5$.

\section{CONCLUSIONS AND OUTLOOK}

We have presented a method to compute a complete Lyapunov function and thus to determine the qualitative behaviour of a given ODE, using both the values of the complete Lyapunov function and its orbital derivative. A complete Lyapunov function $V$ is constant along solutions in the chain-recurrent set, i.e. the 

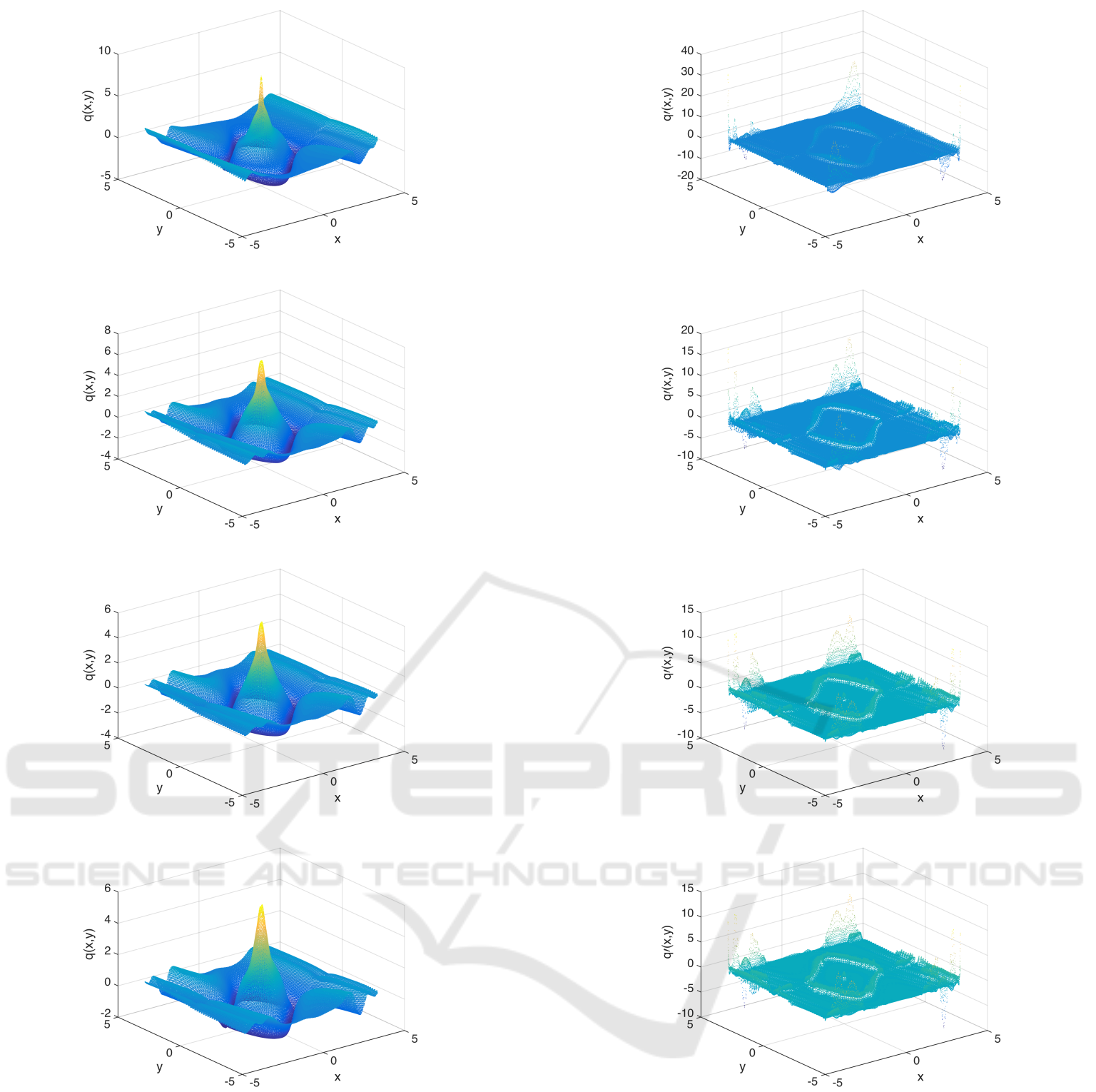

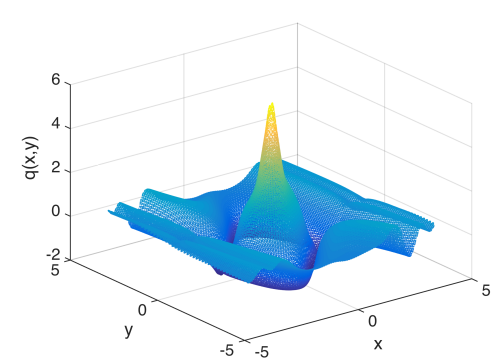

Figure 10: Evolution of the complete Lyapunov function approximation $v(\mathbf{x})$ for the Van der Pol problem with critical value $\gamma=-0.25, \alpha=0.1, r=0.5$. The top figure shows the first iteration of the method, the second, third and fourth show the corresponding iterations. The figures only change very slightly after the first step; one can observe that the range of values is reduced.

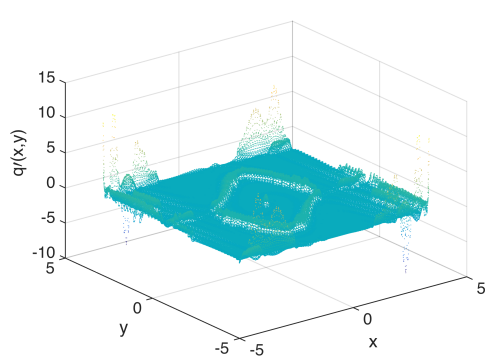

Figure 11: Evolution of the complete Lyapunov function approximation $v^{\prime}(\mathbf{x})$ for the Van der Pol problem for the steps of the method. After the first step, where points with poor approximation have orbital derivatives with positive values, they are close to zero in subsequent steps. There are no significant changes in the later steps. 

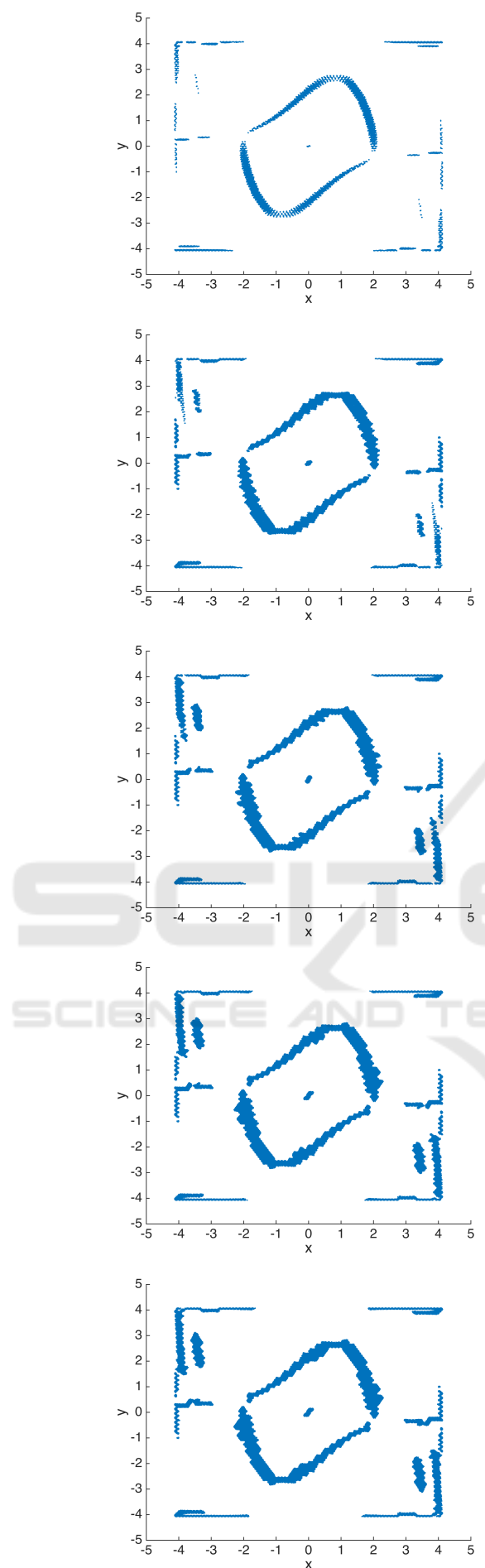

Figure 12: Evolution of the checking points with orbital derivative larger than the critical value $\gamma=-0.25$ for the Van der Pol problem over several steps. It can be seen that after the first step visibly more points are added, which nearly cover the whole periodic orbit, while in later steps there are no significant changes. Apart from the chainrecurrent sets, also points at the boundary of the domain are marked as poor approximations.
Table 1: In this table we list the overall number of collocation points as well as the collocation points in $X^{0}$ in each step of the algorithm for each of the examples (4) to (6). In example (4), no further points are added after step 4, while for the other two examples we keep adding points to $X^{0}$.

\begin{tabular}{|r||r|r|r|}
\hline system & $(4)$ & $(5)$ & $(6)$ \\
\hline collocation points & 29440 & 7708 & 19500 \\
\hline \hline step 1 & 1358 & 762 & 2517 \\
\hline step 2 & 1362 & 956 & 2751 \\
\hline step 3 & 1368 & 1034 & 2802 \\
\hline step 4 & 1370 & 1072 & 2820 \\
\hline step 5 & 1370 & 1092 & 2829 \\
\hline
\end{tabular}

Table 2: Parameters to obtain results shown in table 1.

\begin{tabular}{|r||r|r|r|}
\hline system & $(4)$ & $(5)$ & $(6)$ \\
\hline \hline$\alpha$ & 0.02 & 0.1 & 0.02 \\
\hline$\gamma$ & -0.25 & -0.25 & 0 \\
\hline$r$ & 0.5 & 0.5 & 0.5 \\
\hline
\end{tabular}

orbital derivative is zero $\left(V^{\prime}(\mathbf{x})=0\right)$, and it is strictly decreasing along solutions in the gradient-like set, i.e. $V^{\prime}(\mathbf{x})<0$. Minima of the complete Lyapunov function correspond to attractors and maxima to repellers.

The method approximately solves the PDE $V^{\prime}(\mathbf{x})=-1$ with mesh-free collocation. As this PDE does not have a solution in the chain-recurrent set, we examine where the approximation is poor (the set $X^{0}$ ) and thus can determine the chain-recurrent set. In the subsequent steps of the algorithm we adjust the PDE problem by solving $V^{\prime}(\mathbf{x})=0$ for $x \in X^{0}$ and $V^{\prime}(\mathbf{x})=-1$ elsewhere to approximate a complete Lyapunov function.

While the method works well in several examples to detect the dynamical behaviour, it does not always converge to a fixed set of points in $X^{0}$ and $X^{-}=X \backslash X^{0}$, but keeps on adding points. Furthermore, points at the boundary of the domain are included in $X^{0}$, although they belong to the gradientlike part. The reason could be that we set the orbital derivative to 0 and -1 , rather than a continuous function. Further research will consider replacing this with a more elaborate method, e.g. by replacing the jump function by a smooth function of the distance to the detected chain-recurrent set.

\section{ACKNOWLEDGEMENTS}

First author in this paper is supported by the Icelandic Research Fund (Rannís) grant number 163074052, Complete Lyapunov functions: Efficient numerical computation. Special thanks to Dr. Jean-Claude Berthet for all his good comments and advices on $\mathrm{C}++$. 


\section{REFERENCES}

Anderson, J. and Papachristodoulou, A. (2015). Advances in computational Lyapunov analysis using sum-ofsquares programming. Discrete Contin. Dyn. Syst. Ser. $B, 20(8): 2361-2381$.

Auslander, J. (1964). Generalized recurrence in dynamical systems. Contr. to Diff. Equ., 3:65-74.

Ban, H. and Kalies, W. (2006). A computational approach to Conley's decomposition theorem. J. Comput. Nonlinear Dynam, 1(4):312-319.

Björnsson, J., Giesl, P., and Hafstein, S. (2014a). Algorithmic verification of approximations to complete Lyapunov functions. In Proceedings of the 21st International Symposium on Mathematical Theory of Networks and Systems, pages 1181-1188 (no. 0180), Groningen, The Netherlands.

Björnsson, J., Giesl, P., Hafstein, S., Kellett, C., and Li, H. (2014b). Computation of continuous and piecewise affine Lyapunov functions by numerical approximations of the Massera construction. In Proceedings of the CDC, 53rd IEEE Conference on Decision and Control, Los Angeles (CA), USA.

Björnsson, J., Giesl, P., Hafstein, S., Kellett, C., and Li, H. (2015). Computation of Lyapunov functions for systems with multiple attractors. Discrete Contin. Dyn. Syst. Ser. A, 35(9):4019-4039.

Conley, C. (1978a). Isolated Invariant Sets and the Morse Index. CBMS Regional Conference Series no. 38. American Mathematical Society.

Conley, C. (1978b). Isolated Invariant Sets and the Morse Index. CBMS Regional Conference Series no. 38. American Mathematical Society.

Conley, C. (1988). The gradient structure of a flow i. Ergodic Theory Dynam. Systems, 8:11-26.

Dellnitz, M., Froyland, G., and Junge, O. (2001). The algorithms behind GAIO - set oriented numerical methods for dynamical systems. In Ergodic theory, analysis, and efficient simulation of dynamical systems, pages 145-174, 805-807. Springer, Berlin.

Dellnitz, M. and Junge, O. (2002). Set oriented numerical methods for dynamical systems. In Handbook of dynamical systems, Vol. 2, pages 221-264. NorthHolland, Amsterdam.

Doban, A. (2016). Stability domains computation and stabilization of nonlinear systems: implications for biological systems. PhD thesis: Eindhoven University of Technology.

Doban, A. and Lazar, M. (2016). Computation of Lyapunov functions for nonlinear differential equations via a Yoshizawa-type construction. IFAC-PapersOnLine, 49(18):29 - 34. 10th IFAC Symposium on Nonlinear Control Systems NOLCOS 2016, Monterey, California, USA, 23-25 August 2016.

Giesl, P. (2007). Construction of Global Lyapunov Functions Using Radial Basis Functions. Lecture Notes in Math. 1904, Springer.

Giesl, P. and Hafstein, S. (2015). Review of computational methods for Lyapunov functions. Discrete Contin. Dyn. Syst. Ser. B, 20(8):2291-2331.
Giesl, P. and Wendland, H. (2007). Meshless collocation: error estimates with application to Dynamical Systems. SIAM J. Numer. Anal., 45(4):1723-1741.

Goullet, A., Harker, S., Mischaikow, K., Kalies, W., and Kasti, D. (2015). Efficient computation of Lyapunov functions for Morse decompositions. Discrete Contin. Dyn. Syst. Ser. B, 20(8):2419-2451.

Hafstein, S. (2007). An algorithm for constructing Lyapunov functions. Monograph. Electron. J. Diff. Eqns.

Hsu, C. S. (1987). Cell-to-cell mapping, volume 64 of Applied Mathematical Sciences. Springer-Verlag, New York.

Hurley, M. (1992). Noncompact chain recurrence and attraction. Proc. Amer. Math. Soc., 115:1139-1148.

Hurley, M. (1995). Chain recurrence, semiflows, and gradients. J Dyn Diff Equat, 7(3):437-456.

Hurley, M. (1998). Lyapunov functions and attractors in arbitrary metric spaces. Proc. Amer. Math. Soc. 126:245-256.

Johansen, T. (2000). Computation of Lyapunov functions for smooth, nonlinear systems using convex optimization. Automatica, 36:1617-1626.

Johansson, M. (1999). Piecewise Linear Control Systems. PhD thesis: Lund University, Sweden.

Kalies, W., Mischaikow, K., and VanderVorst, R. (2005). An algorithmic approach to chain recurrence. Found. Comput. Math, 5(4):409-449.

Kamyar, R. and Peet, M. (2015). Polynomial optimization with applications to stability analysis and control - an alternative to sum of squares. Discrete Contin. Dyn. Syst. Ser. B, 20(8):2383-2417.

Krauskopf, B., Osinga, H., Doedel, E. J., Henderson, M., Guckenheimer, J., Vladimirsky, A., Dellnitz, M., and Junge, O. (2005). A survey of methods for computing (un)stable manifolds of vector fields. Internat. J. Bifur. Chaos Appl. Sci. Engrg., 15(3):763-791.

Lyapunov, A. M. (1992). The general problem of the stability of motion. Internat. J. Control, 55(3):521-790. Translated by A. T. Fuller from Édouard Davaux's French translation (1907) of the 1892 Russian original, With an editorial (historical introduction) by Fuller, a biography of Lyapunov by V. I. Smirnov, and the bibliography of Lyapunov's works collected by J. F. Barrett, Lyapunov centenary issue.

Marinósson, S. (2002). Lyapunov function construction for ordinary differential equations with linear programming. Dynamical Systems: An International Journal, 17:137-150.

Narcowich, F. J., Ward, J. D., and Wendland, H. (2005). Sobolev bounds on functions with scattered zeros, with applications to radial basis function surface fitting. Mathematics of Computation, 74:743-763.

Osipenko, G. (2007). Dynamical systems, graphs, and algorithms. Springer, Berlin. Lecture Notes in Mathematics 1889.

Wendland, H. (1998). Error estimates for interpolation by compactly supported Radial Basis Functions of minimal degree. J. Approx. Theory, 93:258-272.

Wendland, H. (2005). Scattered data approximation, volume 17 of Cambridge Monographs on Applied and Computational Mathematics. Cambridge University Press, Cambridge. 